\title{
Hipparcos photometry of 24 variable massive stars ( $\alpha$ Cygni variables)
}

\author{
F. van Leeuwen ${ }^{1}$, A.M. van Genderen ${ }^{2}$, and I. Zegelaar ${ }^{2}$ \\ 1 Royal Greenwich Observatory, Madingley Road, Cambridge, CB3 0EZ, UK \\ ${ }^{2}$ Leiden Observatory, Postbus 9513, 2300 RA Leiden, The Netherlands
}

Received May 28; accepted June 17, 1997

\begin{abstract}
The photometric variability of $24 \alpha$ Cyg variables, i.e. variable super-and hypergiants, observed by the Hipparcos satellite is described. Three of the selected stars are situated in the SMC, 12 in the LMC and the remaining 9 in the Galactic plane. Four of them are hot S Dortype variables, or LBVs (luminous blue variables) and two are possible members of this class. Light curves are presented for all stars. For five stars, among which one LBV, the variability was discovered from the Hipparcos photometric data. Results of period searches are presented and, when relevant, folded light curves are shown. The linear ephemeris of two variables was revised.

For most of the program stars the Hipparcos magnitude scale $(H p)$ differs from the $V$ of the $U B V$ system by $\lesssim 0.1$. For all variables temperatures and $M_{\text {bol }}$ are given. Galactic foreground reddening for the objects in the Magellanic Clouds are given based on IRAS maps.
\end{abstract}

Key words: stars: variables — stars: supergiants techniques: photometric — magellanic clouds

\section{Introduction}

We present the results of an investigation of the one channel $H p$ photometry made by the Hipparcos satellite and presented in the Hipparcos and Tycho Catalogues (ESA, 1997) for 24 variable super-and hypergiants: the $\alpha$ Cyg variables. The observations were obtained between November 1989 and March 1993 and are presented in Fig. 1 to Fig. 3. These massive stars show without exception photometric micro variations, also referred to as $\alpha$ Cyg-type variations, provided that they are evolved and massive enough $\left(\gtrsim 25 M_{\mathrm{i}} / M_{\odot}\right)$. The OB-type stars in this group show brightness variations usually $\lesssim 0$. 1 , the later type stars may show brightness variations of the same order, but often also larger variations. For the early type

Send offprint requests to: F. van Leeuwen stars the typical time scales for the variations is $2-6$ weeks, but for later type stars it can reach a few hundred days (van Genderen 1989, 1991).

Due to their non-strict periodicity, i.e. light curve shapes vary from cycle-to-cycle, the "periods" are in fact "quasi-" or "pseudo-periods". It is quite well possible that the cause for this must be sought, partly or largely, in a multi-periodic origin (van Genderen et al. 1989; Sterken et al. 1997) together with possible effects of additional large scale atmospheric motion fields (e.g. de Jager et al. 1984; Burki 1987; van Genderen 1991) and other stochastic variations (Sterken et al. 1997).

The variables presented here were part of a larger sample, and were selected on account of their reputation and/or their relatively large light variations. This selection contains 3 SMC-, 12 LMC- and 9 Galactic members, of which $4 \mathrm{~S}$ Dor-type variables or LBVs and 2 possible members of this class. For each of these stars a period analysis was performed, the results of which were compared with previous studies if available.

A comparison has been made between the $H p$ magnitudes and magnitudes in the Johnson $V$ band, $V_{\mathrm{J}}$. These were either obtained with a $U B V$ photometer, or based on the transformation to $V_{\mathrm{J}}$ of the Walraven $V$ or Strömgren $y$ band. Observations in the latter system have been obtained by the LTPV project (Long-Term Photometry of Variables) led by Sterken (1983).

\section{The period analyses}

The statistical aspects of the use of the Hipparcos photometric data in variability research have been described by van Leeuwen et al. (1997) and in Vol. 3 of ESA (1997). The distribution of gaps and the lengths of data stretches is far from optimal for periods between a few days and 40 to 70 days, and aliassing is often impossible to distinguish: the window functions resulting from the distribution of data were generally very poor. Statistical indicators of 

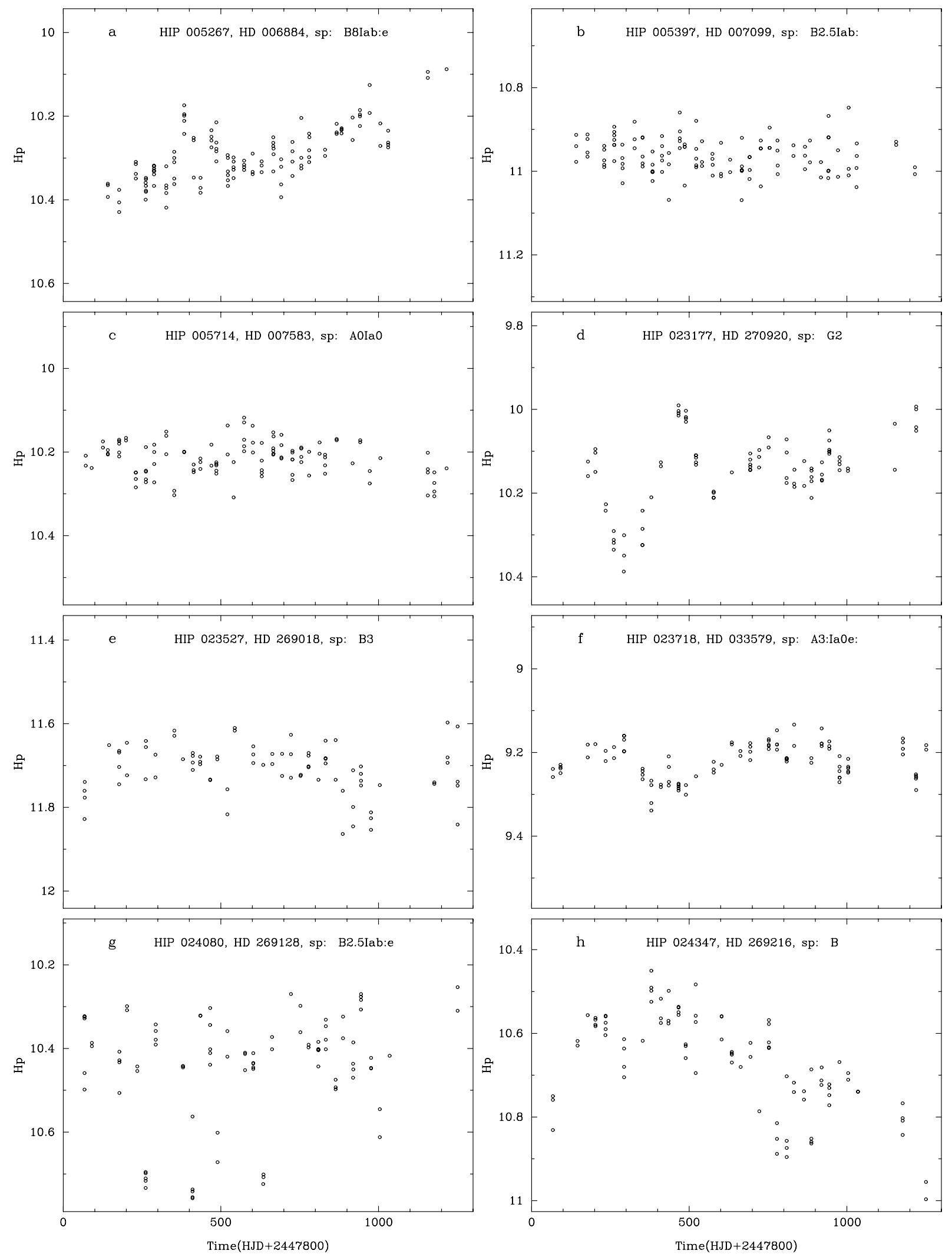

Fig. 1. Light curves for the first 8 stars as $H p$ versus Baricentric Julian Day 

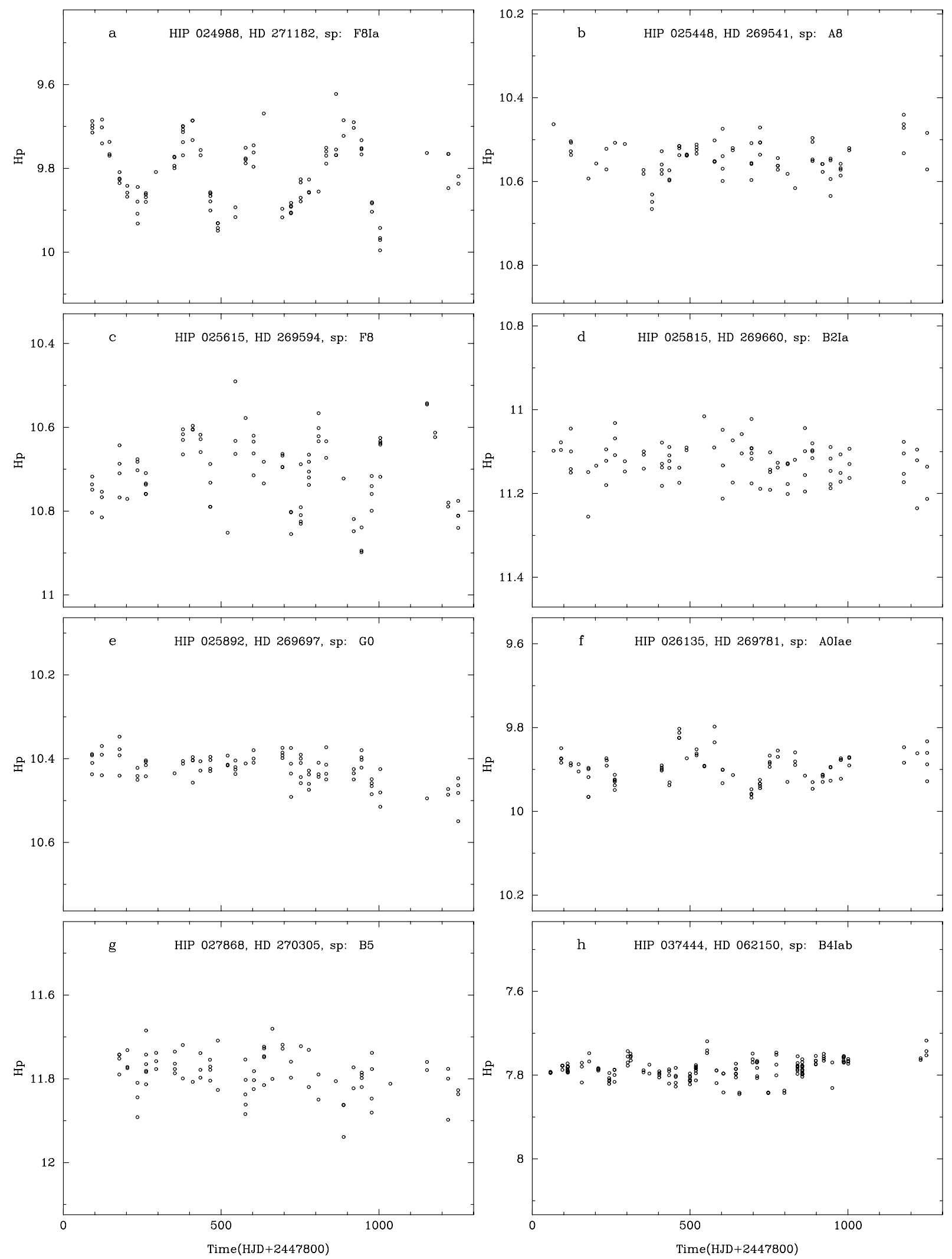

Fig. 2. Light curves for the second group of 8 stars as $H p$ versus Baricentric Julian Day 

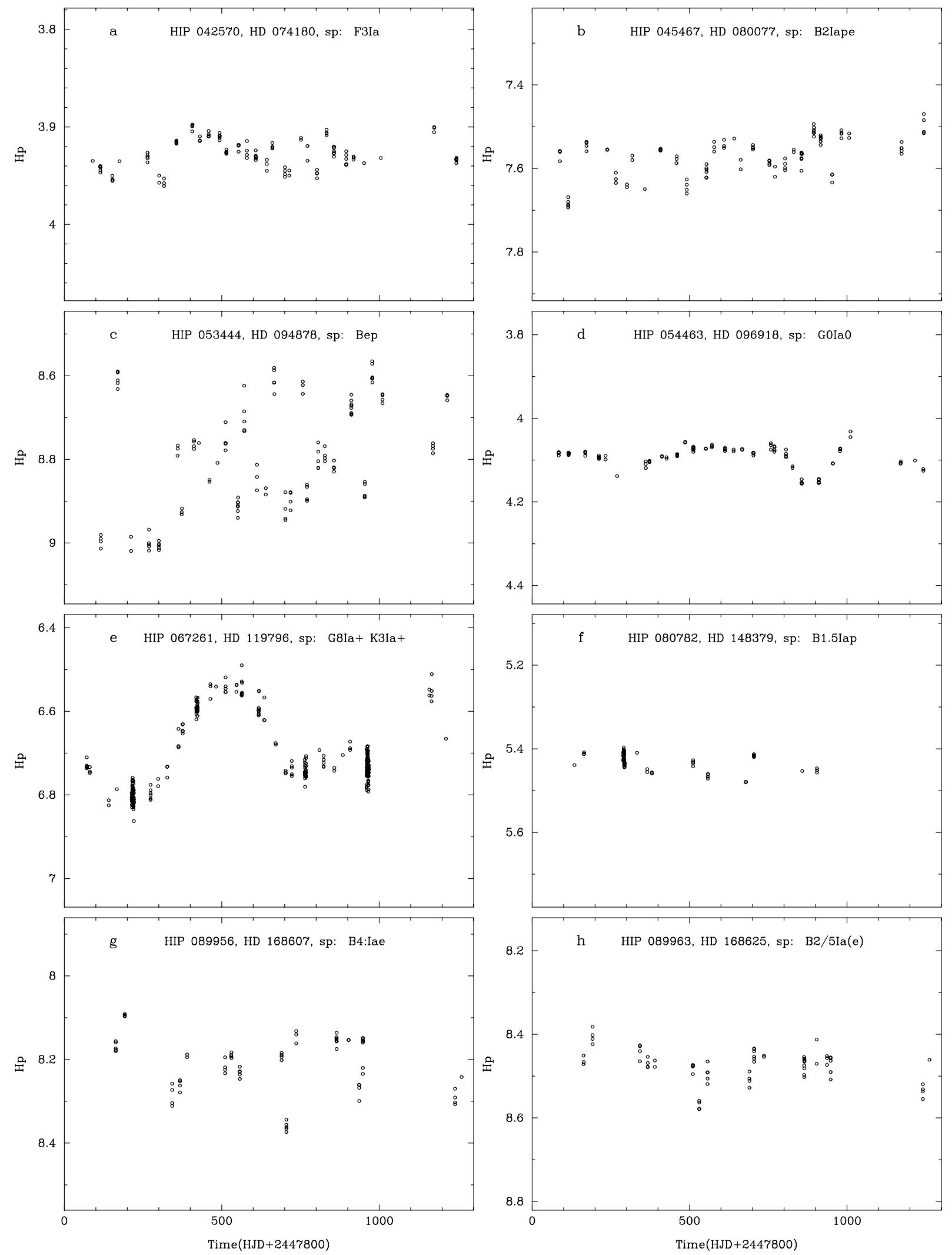

Fig. 3. Light curves for the last group of 8 stars as $H p$ versus Baricentric Julian Day 


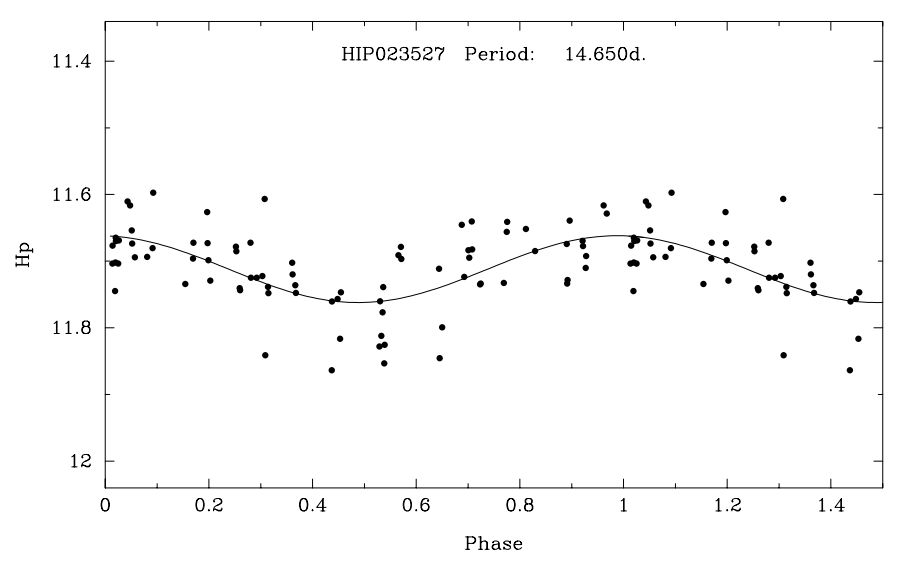

Fig. 4. The phase diagram for HIP $23527=$ HDE 269018

variability were, due to the relatively large number of observations per star, on the other hand quite reliable.

For all stars the period searching method of Stellingwerf (1978) was used. In many cases we also made use of the algorithm of Sterken (1977), which is based on a sine curve fit to the data, usually with more or less the same results. For the early type stars the search was made between $1 \mathrm{~d}$ and $100 \mathrm{~d}$ and extended to a few hundred days for the late type stars.

Because of the difficulty in defining a reliable quasiperiod, we often present a few periods resulting from the periodogram in order of decreasing significance. In some cases, two or more actual periods can be present, like this is the case for $\zeta^{1}$ Sco and R 40 (Sterken et al. 1997, 1998). With the most dominant period one can often obtain a satisfactory phase diagram, which may give the wrong impression of being the only "true" quasi-period, with the remaining scatter only due to different types of stochastic processes. Apparently significant periods are illustrated with folded light curves, but this does not exclude other or alternative periods being possible.

The stars are described in order of increasing HIP identifier. This identifier is then followed by one or more of the following catalogue numbers: HR/HD/HDE number, $\mathrm{R}$ (Radcliffe) number for the Magellanic Cloud objects (Feast et al. 1969), and if available the name given in the General Catalogue of Variable Stars and its extensions (Kholopov et al. 1985). Spectral types used in the text are those we considered the most reliable available in the literature, those in Fig. 1 to Fig. 3 are the ones presented in ESA 1997, except for HIP 67261, where the spectral type as given by ESA 1997 is very wrong (A7V i.s.o. G8Ia).

\section{Discussion on the individual variables}

\subsection{The variables in the $S M C$}

\section{- HIP $5267=$ HD $6884=$ R 40, B9 Iae}

This object is an LBV, discovered by Szeifert et al. (1983), and started a prominent SD (S Dor) phase around 1960, with a steep rise in 1988. The light curve has been described by van Genderen et al. (1997b, Paper I). In a forthcoming paper it will be demonstrated that it is a multi-periodic variable (Sterken et al. 1998). Hipparcos observed the star during the steep rise (Fig. 1a) partly coinciding with the data groups 56-7 and 8-9-10 in Paper I and the gaps in between. After subtraction of the long-term rise, the period search applied to the $H p$ data revealed as best period for the micro variations $84.8 \mathrm{~d}, 86.2 \mathrm{~d}$ and $96.2 \mathrm{~d}$. The last one lies well between the periods for the two groups mentioned above, viz. $93.5 \mathrm{~d}$ and $98.3 \mathrm{~d}$ and supports the increasing trend of the period for this type of micro variations noticed during the rise to maximum brightness of other LBVs (in Paper I they are called the $100 \mathrm{~d}$-type micro variations). The other two periods should then be spurious.

\section{- HIP $5397=$ HD $7099=$ R 42, B3 Ia}

Based on still unpublished $V B L U W$ photometry made between 1987 and 1990, this star is known to be variable with a total range of 0.15 and a time scale of $2-4$ weeks. The $H p$ data show a light range of $0 .{ }^{\mathrm{m}} 11$ (Fig. 1b).

The periodogram of the $H p$ data shows, with little difference in significance, peaks near $15 \mathrm{~d}, 23 \mathrm{~d}$ and $47 \mathrm{~d}$. The periodogram for the unpublished $V$ data, which are much more numerous, show as most significant peaks near 16 d, 16.6 d, 20.6 d and 25.3 d. Evidently, the significance of the $H p$ periods $15 \mathrm{~d}$ and $23 \mathrm{~d}$ are supported by these ones, while the one at $47 \mathrm{~d}$ may be the $3 \mathrm{P}$ or $2 \mathrm{P}$ alias, respectively, of the other two.

- HIP $5714=$ HD $7583=$ R 45, $\mathrm{AO} \mathrm{Ia}^{+}$

Based on still unpublished $V B L U W$ photometry made between 1986 and 1989 this hypergiant is known to be variable with a total range of 0.12 and a time scale of 6-11 weeks. The $H p$ data show a similar light range (Fig. 1c).

The periodogram of the $H p$ data shows a most pronounced peak near $24.8 \mathrm{~d}$ and with less significance peaks near $67 \mathrm{~d}$ and $49 \mathrm{~d}$. The periodogram for the much more numerous unpublished $V$ data shows a number of best periods between $50 \mathrm{~d}$ and $77 \mathrm{~d}$ supporting the last two periods for the $H p$ data. The $24.8 \mathrm{~d}$ $H p$ period probably results from the window function since it is completely absent in the $V$ data.

\subsection{The variables in the $L M C$}

\section{- HIP 23177 = HDE 270920, G2 Ia}

The variability, discovered by Grieve et al. (1985), showed in the past a total range of 0.3 in $V_{\mathrm{J}}$ at a time scale of $250 \mathrm{~d}$. The light curve of the $H p$ data in Fig. 1d shows about 5 cycles with time scales of $100 \mathrm{~d}-300 \mathrm{~d}$ and ranges between $0 .{ }^{\mathrm{m}} 1$ and $0^{\mathrm{m}} \cdot 4$. Thus, the overall behaviour did not change. 

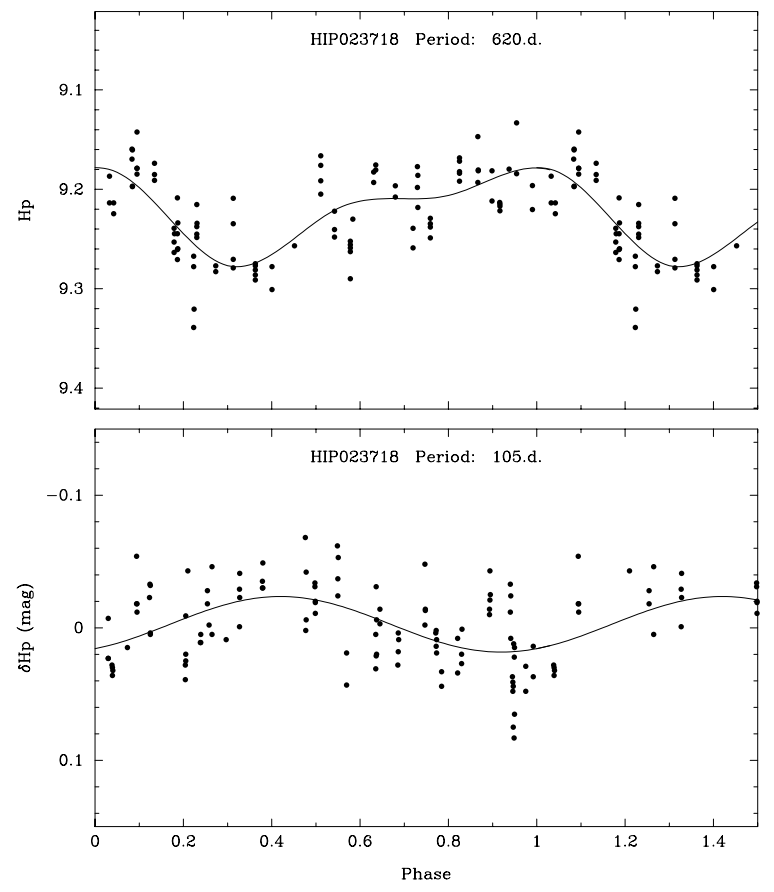

Fig. 5. The phase diagram for the long-time oscilation (top) and the weak short-time scale oscillation of HIP $23718=$ HD $33579=\mathrm{R} 76$. Residuals from the upper curve were used to construct the lower curve

\section{- HIP 23527 = HDE 269018, B2.5 Ia}

The variability has been established by the $H p$ data, showing a range of 0.10 (Fig. 1e). The periodogram shows four candidate periods which in order of decreasing significance are: $14.6 \mathrm{~d}, 6.3 \mathrm{~d}, 32.5 \mathrm{~d}$ and $30.2 \mathrm{~d}$. The phase diagram for the best one is shown in Fig. 4. The third may be the spectral window function and the fourth the $2 \mathrm{P}$ alias of the first period.

- HIP $23718=$ HD $33579=$ R 76, A3 $\mathrm{Ia}^{+}$

The variability of one of the most luminous stars in the LMC has been established by van Genderen (1979). It was characterized by a $100 \mathrm{~d}$ oscillation with a range of 0.05 . Later, it appeared that an underlying and stronger oscillation exists with a presumable time scale of $1-3 \mathrm{yr}$ and a range of 0.1 (Grieve \& Madore 1986). The $H p$ data show for the first time both oscillations, the weak and the strong one, very well. Figure $1 \mathrm{f}$ shows at least two long-time scale cycles of the strong oscillation with a time scale of $\sim 2 \mathrm{yr}$ and a range of 0 . 12 . Figure 5 (top panel) shows the phase diagram for a $620 \mathrm{~d}$ period. The periodogram for the weak oscillation with a total range of 0.03 , derived from the scatter around the mean light curve, reveals a number of peaks which in order of decreasing significance are: $105 \mathrm{~d}, 81 \mathrm{~d}, 57 \mathrm{~d}$ and $70 \mathrm{~d}$. Since the first one is similar to the quasi-period found earlier which was based on almost $5 \mathrm{yr}$ of monitoring, we presume that this is the true period. Figure 5 (bottom panel) shows the phase diagram. However, it must be emphasized that the way of drawing the mean curve through the strong oscillation is not completely objective, though we do not expect much influence on the periodogram.

The importance of the $H p$ light curve lies in the fact that both oscillations can be seen simultaneously, the weak one superimposed on the strong one. This is a support for the study of the atmospheric dynamics with the aid of spectra by Nieuwenhuijzen et al. (1997), who have identified both types of oscillations in a spectroscopic data set obtained in 1986 and 1987.

- HIP 24080 = HDE $269128=$ R 81, B2.5 Iab

Based on Strömgren photometry, made by the LTPV group, Stahl et al. (1987) discovered that this LBV is also an eclipsing binary with a period of $74.59 \mathrm{~d}$. $\alpha$ Cyg-type variations are superimposed on the light curve. The depth of the primary minimum amounts to $0 \mathrm{~m} 4$. No secondary minimum is present. For the $\alpha$ Cyg-type variations a quasi-period was established amounting to $24 \mathrm{~d}$ (van Genderen et al. 1992).

By collecting the eclipses seen in the $H p$ data (Fig. 1g) and in the LTPV data (Manfroid et al. 1994, Sterken et al. 1993, 1995), it became clear that the linear ephemeris by Stahl et al. (1987) needed a small revision. A new linear ephemeris was determined by selecting all observations close to mid eclipse, i.e. with $H p$ and $V_{\mathrm{J}} \geqq 10.7$. In this way we extracted from the LTPV catalogues and the $H p$ data 30 and 3 observations, respectively. In total the time base amounts to 44 cycles. The least-squares solution resulted in the following relation:

$$
\begin{gathered}
\mathrm{JD}_{\text {min }}=2445973.48+74.55 \times E \\
\pm 0.40 \pm 0.02 .
\end{gathered}
$$

The plot of the $(\mathrm{O}-\mathrm{C})$ values is shown in Fig. 6. No search for a period for the $\alpha$ Cyg variations at maximum light was undertaken. The reason is that the number of observations is too low. Besides, the light curve is complicated. Instead of a secondary minimum, the light curve only shows a steady decline between successive primary minima (see Stahl et al. 1987).

\section{- HIP 24347 = HDE 269216, B8 I}

This object has spectroscopically a strong resemblance with LBVs near minimum light such as HDE 269006 $=\mathrm{R} 71$ (Prinja \& Schild 1991). Indeed, the $H p$ light curve in Fig. 1h strongly supports their suspicion as will be explained below. The light curve shows two long-time scale cycles amounting to $700 \mathrm{~d}$ and $500 \mathrm{~d}$ and a range of 0.4 with superimposed $\alpha$ Cyg-type variations with a range of 0.1 and a time scale of the order of weeks (see below). This estimated time scale is based on the analysis of the scatter around the mean curve sketched through the two cycles and is therefore not completely objective. The periodogram revealed a few peaks near the following periods in order of decreasing significance: $16.1 \mathrm{~d}, 20.5 \mathrm{~d}, 8.1 \mathrm{~d}$ and 
$9.9 \mathrm{~d}$. The latter two may be the $1 / 2 \mathrm{P}$ aliases of the first two periods. Figure 7 shows the phase diagram for $16.1 \mathrm{~d}$.

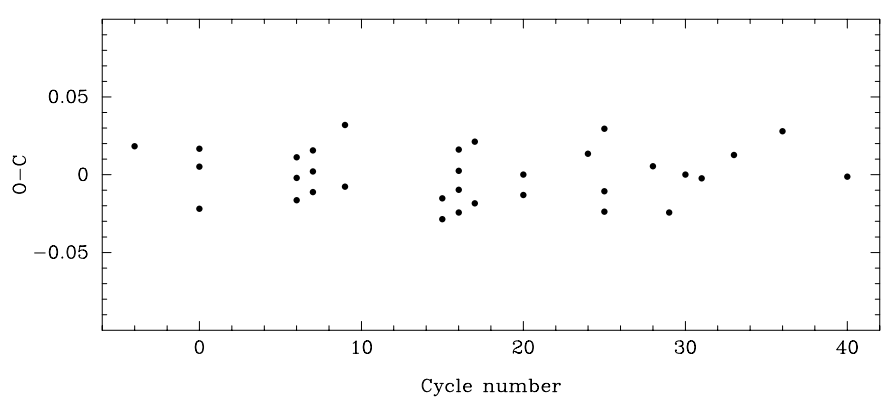

Fig. 6. The $(\mathrm{O}-\mathrm{C})$ versus cycle number $\mathrm{E}$ of HIP $24080=$ HDE $269128=\mathrm{R} 81$ based on the binary period amounting to $74.55 \mathrm{~d}$

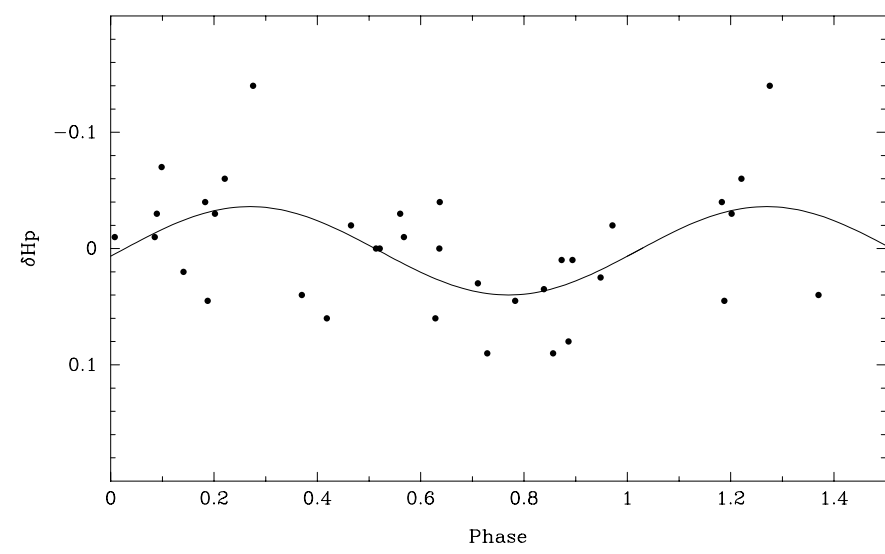

Fig. 7. The phase diagram with $P=16.1 \mathrm{~d}$ for the short-time scale oscillation of HIP $24347=$ HDE 269216. Residuals read from a smooth curve through the long-time scale oscillation in units of $0 .{ }^{\mathrm{m}} 01$

The resemblance of the light variation with that of R 71 is striking. The latter showed between 1970 and 1980 an enhanced apparent brightness of $\sim 1 \mathrm{~m}$. Subsequently, two, but much weaker active states occurred lasting 5 and $3 \mathrm{yr}$ and with ranges of a few 0 . 1 . The latter are roughly similar to the two cycles of our program star. According to the new nomenclature introduced by van Genderen et al. (1997a), these episodes of enhanced brightness are called "normal S Dor (SD) phases".

Superimposed on these episodes, R71 also shows $\alpha$ Cyg -type variations in the order of $2-4$ weeks (e.g. van Genderen et al. 1997b). All these characteristics in common support the suspicion that HDE 269216 is indeed an LBV.

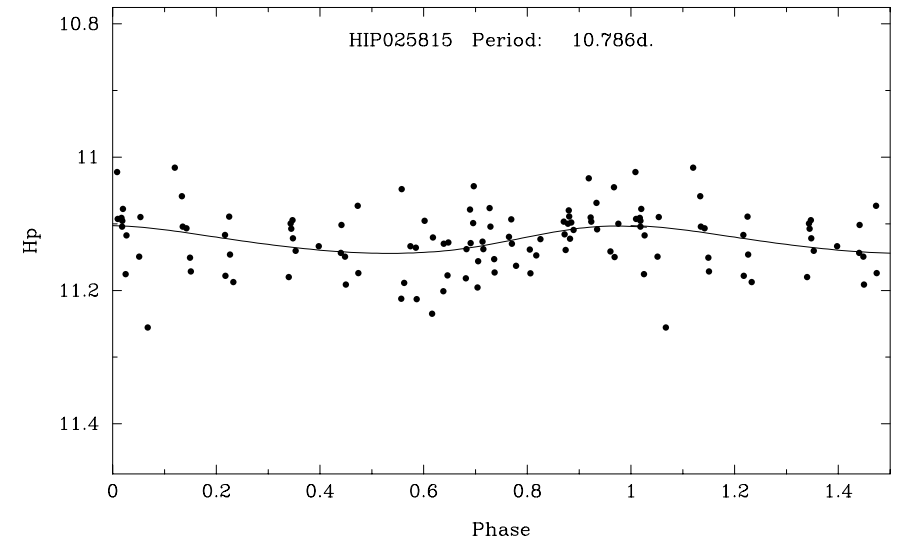

Fig. 8. The phase diagram for HIP $25815=$ HDE $269660=$ R 112

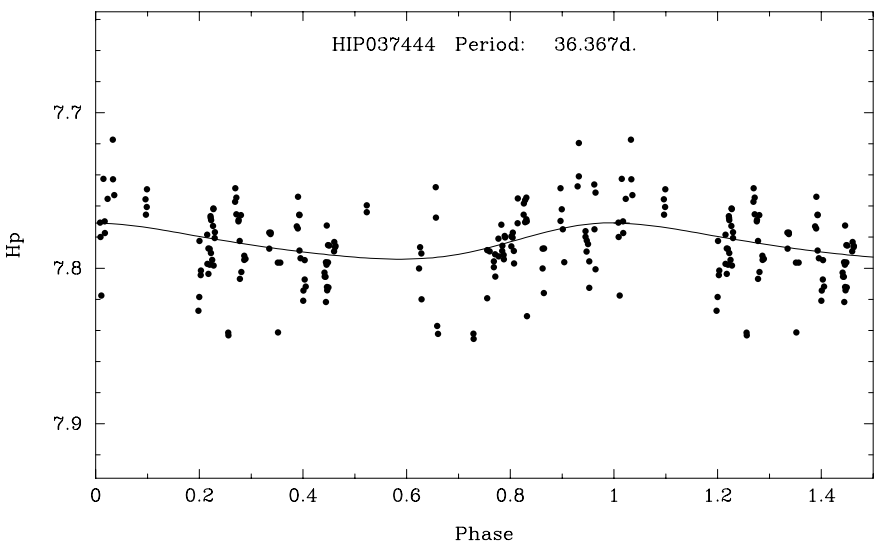

Fig. 9. The phase diagram for HIP $37444=$ HD 62150

\section{- HIP $24988=$ HDE $271182=$ R 92, F8 Ia ${ }^{+}$}

The variability found by Eggen (1983), has been confirmed by Grieve et al. (1985), van Genderen et al. (1986) and van Genderen \& Hadiyanto (1989). The light curve showed oscillations amounting to $200 \mathrm{~d}$ to $250 \mathrm{~d}$, with a range of a few 0.1 . The $H p$ light curve behaves similarly (Fig. 2a). There are almost five cycles with a time scale of $260 \mathrm{~d}$. The extrema of the brightness hover between $H p=9.7$ and 10, which is fainter by $\sim 0.2$ than the $V_{\mathrm{J}}$ magnitudes from the references quoted above: $V_{\mathrm{J}}=9.6-9.7$. This difference is larger than any of the $\delta m=H p-V_{\mathrm{J}}$ value listed in Tables $1-3$. For the minima of the oscillations the difference is even 0.3 . Therefore, it might be that still another, much longer oscillation is present.

- HIP 25448 = HDE 269541, A8: $\mathrm{Ia}^{+}$

The variability, with unknown time scale, was first detected by Grieve \& Madore (1986). The magnitude limits for $V_{\mathrm{J}}$ were 10.40 and 10.57 . The $H p$ light curve (Fig. 2b) confirms the variability, but with a lower range of $0 .{ }^{\mathrm{m}} 1$. The periodogram shows a few peaks which in order of decreasing significance are: $8.1 \mathrm{~d}$, 
$24.6 \mathrm{~d}, 12.0 \mathrm{~d}, 40.5 \mathrm{~d}$ and $27.0 \mathrm{~d}$. The last one is presumably the result of the spectral window function. The first period, although the most significant one, is not compatible (as well as the other ones) with the empirical evidence that such late type star should have much longer quasi-periods say $\gtrsim 100 \mathrm{~d}$ (e.g. Burki 1976; Sterken 1977). The periodogram between $100 \mathrm{~d}$ and $400 \mathrm{~d}$ reveals peaks (but with a significance comparable to those mentioned above, but much less than for $8.1 \mathrm{~d}$ ) near $182 \mathrm{~d}$ and $146 \mathrm{~d}$. We cannot claim any conclusion based on these results.

- HIP 25615 = HDE 269594, F8 Ia

The variability was suspected by van Genderen et al. (1982) and Grieve \& Madore (1986). The last mentioned authors also detected the largest range: 0.19 and a possible time scale of $\sim 1$ yr. The $H p$ light curve shows for the first time how the variability looks like: four consecutive oscillations with a time scale of $\sim 200 \mathrm{~d}$ and a range even up to 0.26 (Fig. 2c).

- HIP $25815=$ HDE $269660=$ R 112, B2 Ia

The $H p$ light curve demonstrates for the first time that the star is variable with a range of 0.1 (Fig. $2 \mathrm{~d}$ ). The periodogram shows a few peaks with little difference in significance: $3.656 \mathrm{~d}, 10.79 \mathrm{~d}$ and $5.390 \mathrm{~d}$. Arbitrarily we have chosen the second period for the phase diagram in Fig. 8. If this is the real period, then the first and third period are the $1 / 3 \mathrm{P}$ and $1 / 2 \mathrm{P}$ aliases of $10.81 \mathrm{~d}$.

\section{- HIP 25892 = HDE 269697, F5 Ia}

Although included in a number of photometric programs performed between 1970 and 1990 (e.g. Grieve \& Madore 1986), no significant light variations were noticed. The $H p$ light curve clearly demonstrates for the first time that the star is variable with a range of 0. 1 (see Fig. 2e). The long-time scale sloping trend of the brightness downward, may be an artifact of statistical effects. It is not caused by the use of a wrong colour in the Hipparcos reductions: the colour used was within $0.01 \mathrm{mag}$ from the correct value.

The periodogram for $1 \mathrm{~d}-200 \mathrm{~d}$, showing a lot of noise below $100 \mathrm{~d}$, revealed two nearly equally significant peaks for $48.0 \mathrm{~d}$ and $84 \mathrm{~d}$. The mean curve in the phase diagrams for both ones have an amplitude of 0.05 and a scatter of the same amount. No decision can be made which of these two is best.

\section{- HIP 26135 = HDE 269781 = R 118, B9 Ia}

This star was suspected variable by Rosendhal \& Snowden (1971) and van Genderen et al. (1982). Only the $H p$ data demonstrate the variability unambiguously with a range of 0.14 (Fig. $2 \mathrm{f}$ ). The periodogram revealed as the most significant peak a period near $39.0 \mathrm{~d}$, of which the phase diagram looks very acceptable. A few less significant peaks appeared between $20 \mathrm{~d}$ and $32.0 \mathrm{~d}(21.8 \mathrm{~d}, 24.4 \mathrm{~d}, 27.2 \mathrm{~d} 31.2 \mathrm{~d}$, possibly a few of them are related to the window function) and near $93.0 \mathrm{~d}$ and $61.0 \mathrm{~d}$.

\section{- HIP 27868 = HDE 270305, B3/5 Ia}

The $H p$ light curve demonstrates for the first time its variability (Fig. $2 \mathrm{~g}$ ). The brightness shows a gradual decline amounting to 0.1 during the three years of observing. Due to the faintness of the object it is impossible to say whether also a real short-term variation is present. The mean $H p$ magnitude amounts to 11.79 and the only magnitude known so far amounts to $V_{\mathrm{J}}=$ 11.73 by Ardeberg et al. (1972). Thus, the difference is small.

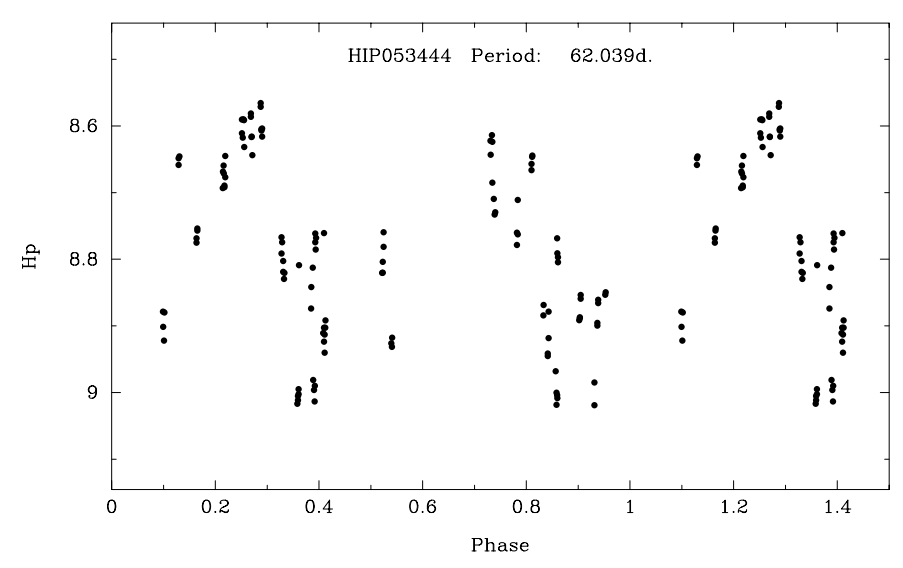

Fig. 10. The phase diagram for HIP $53444=$ HD $94878=$ GG Car

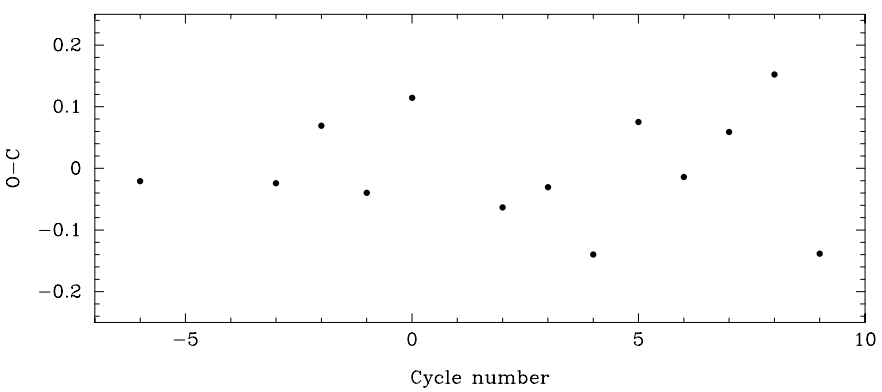

Fig. 11. The $(\mathrm{O}-\mathrm{C})$ in fractions of a cycle versus cycle number $\mathrm{E}$ of HIP $67261=$ HR 5171A $=$ HD $119796=$ V766 Cen

\subsection{The variables in the Galaxy}

\section{- HIP $37444=$ HD 62150, B4 Ia}

The variability is known and based on $V B L U W$ photometry. Considering the time scale of long strings of observations, the period could not be smaller than say $20 \mathrm{~d}$. A possible quasi-period of $45.0 \mathrm{~d}$ was suggested by van Genderen et al. (1985) and van Genderen (1985). Figure $2 \mathrm{~h}$ shows the light curve for the Hipparcos observations. 


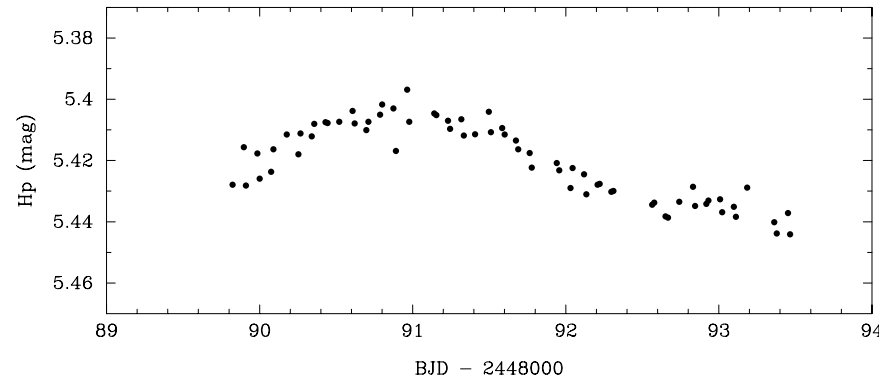

Fig. 12. The monitored part of the light curve of HIP $80782=$ HD $148379=$ QU Nor with a time resolution of $\sim 1 \mathrm{~h}$

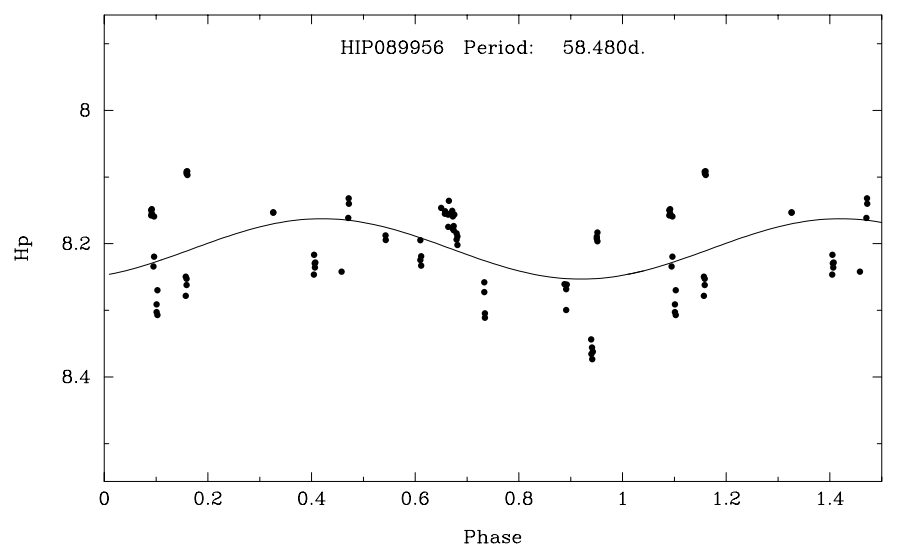

Fig. 13. The phase diagram for HIP $89956=$ HD $168607=$ V4029 Sgr

The periodogram of the $H p$ data revealed only a small peak near $43.0 \mathrm{~d}$, while near $36.4 \mathrm{~d}$ a much stronger peak is present. Further, there are a few peaks close around $73 \mathrm{~d}$, which we consider as the $2 \mathrm{P}$ aliases of $36.4 \mathrm{~d}$. Figure 9 shows the phase diagram for $36.4 \mathrm{~d}$.

- HIP $42570=$ HD74180, F2 Ia ${ }^{+}$

The variability was discussed by Steemers \& van Genderen (1986), but the number of observations was too low to find a reliable quasi-period. It could be somewhere close to 3 months. Also the range of 0.15 was not reliable. Unpublished data made by the LTPV group between JD 2448646 and JD 2449400 , comprising 23 data points, contain a few strings of observations excluding cycle lengths shorter than say $40 \mathrm{~d}$.

The $H p$ light curve (Fig. $3 \mathrm{a}$ ) shows a range of $0 \mathrm{~m} 06$. The periodogram for $P$ longer than $40 \mathrm{~d}$ revealed peaks near $53 \mathrm{~d}, 80 \mathrm{~d}$ and $160 \mathrm{~d}$. The latter may be the $3 \mathrm{P}$ and $2 \mathrm{P}$ alias of the first two periods, respectively.

- HIP $45467=$ HD 80077, B2/3 $\mathrm{Ia}^{+}$

The variability has been discussed by van Genderen et al. (1992), who suggested as possible quasi-periods $41.5 \mathrm{~d}$ or $55.5 \mathrm{~d}$. Long strings of observations exclude periods much shorter than these ones. According to Carpay et al. $(1989,1991)$ the star could be an LBV. The relative large light range, viz. 0.2 , supports that
Table 1. A comparison of the $H p$ magnitudes with nearly simultaneous observations $V_{\mathrm{J}}$

\begin{tabular}{llrll}
\hline \multicolumn{1}{c}{ Star } & \multicolumn{1}{c}{ Sp } & \multicolumn{1}{c}{$\delta m$} & $n$ & Notes \\
\hline HIP 05267 = R 40 & B9 Iae-F Iae & 0.02 & 7 & \\
HIP 05397 = R 42 & B2.5/3 Ia & -0.05 & 2 & 1 \\
HIP 24080= R 81 & B2.5 Iab & -0.04 & 1 & \\
HIP 42570= HD 74180 & F2 Ia & 0.13 & 1 & 1 \\
HIP 45467 = HD 80077 & B2/3 Ia & 0.07 & 3 & 2 \\
HIP 54463 = V382 Car & G0 Ia $^{+}$ & 0.18 & 4 & 3 \\
HIP 67261 = V766 Cen & G8-K3 Ia $^{+}$ & -0.15 & & 4 \\
HIP 89956 = V4029 Sgr & B9 Ia $^{+}$ & 0.04 & 4 & 2 \\
HIP 89963 = V4030 Sgr & B5/8 Ia $^{+}$ & 0.08 & 4 & 2 \\
\hline
\end{tabular}

Notes to Table 1:

${ }^{1}$ Unpublished Walraven $V B L U W$ and /or Strömgren uvby photometry.

${ }^{2}$ van Genderen et al. (1992).

3 Achmad et al. (1992).

${ }^{4}$ Comparison based on overlapping parts of the light curve (van Genderen 1992).

suggestion as well as the possible presence of a shell (Houk 1978).

The $H p$ data, shown in Fig. 3a, partly overlap with the observations made by van Genderen et al. (1992) in the Walraven $V B L U W$ system, which also show a light range of 0.2 . In order to make a combined data set, the $H p$ magnitudes were transformed to $V$. At three occasions observations in both photometric systems were made nearly simultaneously (within $2 \mathrm{~d}$ ). It appeared that the difference $\delta m=H p-V_{\mathrm{J}}=0.07$ (see Table 1; $V_{\mathrm{J}}$ is the $V$ of the $U B V$ system transformed from the $V$ of the Walraven system). The periodogram of the combined data set revealed as most significant peaks those near $66.5 \mathrm{~d}$ and $55.5 \mathrm{~d}$ and with much less significance peaks near $76.0 \mathrm{~d}$ and $41.4 \mathrm{~d}$. The second and fourth period were the most significant ones for the $V$ data alone. The inclusion of the $H p$ data has shifted the highest significance to $66.5 \mathrm{~d}$. However, its phase diagram as well as that for $55.5 \mathrm{~d}$ period, show close to the phase for maximum light two low-brightness data points (one $V$ and one $H p$ point) representing the extreme deep minimum near JD 2447915 (see the light curve in van Genderen et al. 1992, Fig. 2). Therefore, we cannot arrive at a conclusion concerning these two periods.

- HIP 53444 = HD 94878 = GG Car, Be

This peculiar variable is probable a complicated binary with an eccentric orbit and of which one of the components is an intrinsic variable (Gosset et al. 1984, 1985). These authors write that no precise period can actually be derived from the radial velocity data. They take tentatively the single value as the best, viz. $31.020 \mathrm{~d}$, 
but if it is a binary indeed, the real one should be $62.039 \mathrm{~d}$.

The light curve for the $H p$ data is shown in Fig. 3c. The periodogram of the $H p$ data confirm that the shortest of the two periods mentioned above has slightly more significance than its double. Yet the phase diagram for the former shows larger scatter at constant phase, sometimes up to 0.2 , than for the latter. Figure 10 shows the phase diagram for the long period. The reason for the larger scatter mentioned above is that the two extrema of the double waved light curve are different in height, supporting the long period. This phenomenon has also been noticed by Gosset et al. (1984). The remaining part of the scatter, which is still $\sim 0 \mathrm{~m} 1$, must be caused by the variability of at least one of the components.

\section{- HIP $54463=$ HD $96918=$ V382 Car, G0 Ia ${ }^{+}$}

Although this hypergiant was suspected to be variable on a long time scale with a range of $\sim 0$. 05 (van Genderen et al. 1986; Achmad et al. 1992), the $H p$ data show for the first time how the light curve looks like (Fig. 3d): almost three cycles of varying shape and range. The largest range observed amounts to 0.12 . The time scale amounts to $1-2 \mathrm{yr}$ and the best phase diagram is obtained with $556 \mathrm{~d}$. No short-time scale variations are evident. Therefore, it is not surprising that a string of observations made in a short time interval suggested constancy (Berdnikov \& Turner 1995).

- HIP 67261 = HR 5171A = HD $119796=$ V766 Cen, G8-K3 $\mathrm{Ia}^{+}$

The photometric history has been discussed by van Genderen 1992 (Paper II). The star shows in the interval 1973-1991 a stable period of $494 \mathrm{~d}$. The $H p$ light curve (Fig. 3e) shows almost three cycles of varying shape and range. The largest range observed amounts to 0.3 . The rising branch of the first cycle is in common with the cycle belonging to maximum 17 (JD 2448320, $E=8$ ) of the light curve in Paper II.

The $H p$ data was affected by the use of a wrong colour index in the data reductions, in fact, the main catalogue gives as spectral type AV. As a result, there is a slope in the $H p$ data as well as a systematic offset with the ground-based data. At the time of submitting this paper, the official correction equations had not been released yet.

Between the two high amplitude cycles, there is a low one at JD 2448670 , which we considered as maximum 18 with $E=9$. The long-time scale light curve discussed in Paper II also shows in visual light such low amplitude cycles (e.g. maximum 15), which appeared to be much more pronounced in the ultraviolet and therefore considered real.

The next high amplitude cycle is maximum $19(E=$ 10 ), of which the precise epoch could not be determined due to the lack of observations. It should lie between JD 2448890 and JD 244 9220. The least-squares solution based on the epochs listed in Table 3 of Paper II and the two new ones, thus, between $E=-6$ and 9 (omitting $E=-8,-7$, see below, and $E=10$ ) resulted in practically the same linear ephemeris as in Paper I:

$\mathrm{JD}_{\max }=2444293( \pm 15)+494 \mathrm{~d}( \pm 3 \mathrm{~d}) \times E$.

Mean errors are given. Thus, the new maxima support the stability of the period during this time interval. Figure 11 shows the $(\mathrm{O}-\mathrm{C})$ values versus the cycle number $E$. The $(\mathrm{O}-\mathrm{C})$ value for cycle number $E=10$ lies between $-13 \mathrm{~d}$ and $-343 \mathrm{~d}$.

The early maxima at $E=-8$ and -9 indicated that the period was much shorter at that time. (It should be noted that the $(\mathrm{O}-\mathrm{C})$ values for these two maxima are listed in Table 3 of Paper II as negative values instead of positive as they should).

\section{- HIP 80782 = HD 148379 = QU Nor, B2 Iab}

The variability has been found by Feinstein (1968) and a number of other investigators listed by van Genderen (1986, Paper III). The quasi-periodicity derived in the latter paper possibly amounts to $13.35 \mathrm{~d}$.

There are only a small number of days with $H p$ data (Fig. 3f), but during five consecutive days the star has been monitored with a time resolution of $\sim 1 \mathrm{~h}$ comprising 69 observations. The phase diagram for all these data do not contradict the suggested quasiperiod above, but to state that it is a confirmation would be exaggerated. Anyway, long strings of observations discussed in Paper III seem to exclude periods shorter than say $10 \mathrm{~d}$. A period search with the present $H p$ material has no sense due to the low number of stretches of observations.

The long series of 69 observations are plotted in Fig. 12 as a function of BJD, showing a smooth trend with a scatter amounting to 0.05 . The trend confirms the photometry of Paper III that data sets a few hours apart can show significant variations of the order of a few percent. These data would cover a phase interval of 0.27 if the period of $13.57 \mathrm{~d}$ is real.

\section{- HIP 89956 = HD $168607=$ V4029 Sgr, B9 Ia}

This hypergiant and LBV near quiescence, exhibits $\alpha$ Cyg-type variations discovered by Sterken (1977). Van Genderen et al. (1992, Paper IV), combining various data sets comprising $18 \mathrm{yr}$, found a possible quasiperiod of $58.48 \mathrm{~d}$. The range amounts to $0{ }^{\mathrm{m}} 3$.

The number of $H p$ data (Fig. $3 \mathrm{~g}$ ) is too low, the overlap with the data sets mentioned above too small and the gaps in time of the $H p$ data too large to initiate a combined period analysis. Figure 13 shows the phase diagram for the $H p$ data constructed with the linear formula for the maxima presented in Paper IV. Evidently, maximum light lies between phase .1 and .6 , while it should lie at phase .0. Thus the period certainly needs a revision, but this cannot be done with the present material. 
Table 2. Particulars of the program stars in the SMC and LMC

\begin{tabular}{|c|c|c|c|c|c|c|c|c|c|c|c|c|}
\hline & Star & & $\mathrm{Sp}$ & $E(B$ & $\bar{V})_{\mathrm{J}}$ & mea & mag & $\delta m$ & range & $\log T_{\text {eff }}$ & $M_{\mathrm{bol}}$ & Remarks \\
\hline HIP & HD & $\mathrm{R}$ & & gal.frgr. & adopt. & $H p$ & $V_{\mathrm{J}}$ & & $(\mathrm{m})$ & & & \\
\hline$\overline{5267}$ & 6884 & 40 & B9 Iae-F Iae & 0.09 & $0.14^{a}$ & & & $b$ & & $4.00-3.94$ & $-9.5--9.7$ & LBV \\
\hline 5397 & 7099 & 42 & B2.5/3 Ia & 0.08 & $0.12^{c}$ & 10.96 & 11.00 & $-0.04^{b}$ & 0.19 & 4.20 & -9.8 & \\
\hline 5714 & 7583 & 45 & $\mathrm{~A} 0 \mathrm{Ia}^{+}$ & 0.07 & $0.15^{c}$ & 10.21 & 10.17 & 0.04 & 0.12 & 3.96 & -9.7 & \\
\hline 23177 & 270920 & & G2 Ia & 0.08 & $0.24^{d}$ & 10.13 & 9.99 & 0.14 & 0.40 & 3.725 & -9.5 & \\
\hline $23527^{e}$ & 269018 & & B2.5/3 Ia & 0.16 & 0.16 & 11.70 & 11.66 & 0.04 & 0.10 & 4.22 & -8.7 & \\
\hline 23718 & 33579 & 76 & $\mathrm{~A} 3 \mathrm{Ia}^{+}$ & 0.12 & & 9.22 & 9.15 & 0.07 & 0.10 & $3.90^{f}$ & $-9.75^{f}$ & \\
\hline 24080 & 269128 & 81 & B2.5 Iab & 0.13 & $0.15^{g}$ & $10.40^{h}$ & $10.47^{h}$ & $-0.07^{b}$ & $0.20^{i}$ & 4.30 & -10.4 & LBV \\
\hline $24347^{e}$ & 269216 & & B8 I & 0.12 & $0.12^{j}$ & 10.70 & 10.78 & & $0.10^{k}$ & 4.09 & -9.0 & $\mathrm{LBV}^{e}$ \\
\hline 24988 & 271182 & 92 & $\mathrm{~F} 8 \mathrm{Ia}^{+}$ & 0.10 & $0.10^{l}$ & 9.80 & 9.7 & & 0.30 & 3.72 & -9.4 & \\
\hline 25448 & 269541 & & $\mathrm{~A} 8 \mathrm{Ia}^{+}$ & 0.06 & $0.07^{m}$ & 10.55 & 10.46 & 0.09 & 0.17 & 3.86 & -8.4 & \\
\hline 25615 & 269594 & & F8 Ia & 0.12 & 0.12 & 10.71 & 10.55 & 0.16 & 0.26 & 3.78 & -8.5 & \\
\hline $25815^{e}$ & 269660 & 112 & $\mathrm{~B} 1.5 / 2 \mathrm{Ia}$ & 0.14 & 0.14 & 11.13 & 11.17 & & 0.11 & 4.25 & -9.3 & \\
\hline $25892^{e}$ & 269697 & & F5 Ia & 0.12 & 0.12 & 10.43 & 10.35 & & 0.10 & 3.82 & -8.6 & \\
\hline 26135 & 269781 & 118 & B9 Ia & 0.11 & $0.15^{n}$ & 9.90 & 9.90 & 0 & 0.14 & 4.01 & -9.7 & \\
\hline $27868^{e}$ & 270305 & & B3/5 Ia & 0.10 & 0.10 & 11.79 & 11.73 & & 0.10 & 4.16 & -8.2 & \\
\hline
\end{tabular}

notes to Table 2:

a Szeifert et al. (1993).

${ }^{c}$ Derived from $\mathrm{Sp} /(B-V)$ Jo relation.

${ }^{b}$ See comparison in Table 1.

${ }^{e}$ Variability discovered by Hipparcos. $\quad{ }^{f}$ Nieuwenhuijzen et al. (1997).

${ }^{g}$ Wolf et al. (1981).

${ }^{i}$ Eclipse depth $0 .{ }^{\mathrm{m}} 4$.

${ }^{k}$ Range normal SD phases $0 .{ }^{\mathrm{m}} 35$.

${ }^{m}$ Grieve \& Madore (1986).

${ }^{h}$ Outside eclipse value.

${ }^{j}$ Prinja \& Schild (1991) used 0. 08.

${ }^{l}$ van Genderen \& Hadiyanto (1989) used 0. 09.

${ }^{n}$ van Genderen et al. (1983).

- HIP $89963=$ HD $168625=$ V4030 Sgr, B5/8 $\mathrm{Ia}^{+}$ This object is presumably an LBV near quiescence according to Nota et al. (1996). It shows $\alpha$ Cyg-type variations discovered by Sterken (1977). Van Genderen et al. (1992, Paper IV) discussed various data sets and found a possible quasi-period lying between $33 \mathrm{~d}$ and $37 \mathrm{~d}$. The range amounts to 0.15 .

Because of the same reasons as for the previous variable, a combined period analysis of the partly overlapping data sets of Paper IV and the $H p$ data (Fig. 3h) could not be undertaken. The periodogram of the $H p$ data alone, although presumably not of much weight, resulted in strong peaks near $39.4 \mathrm{~d}, 32 \mathrm{~d}$ and the presumably $2 \mathrm{P}$ aliases $79 \mathrm{~d}$ and $65 \mathrm{~d}$, respectively. Therefore, we consider this result as a tentative support of the result presented in Paper IV that the quasiperiod should be $\sim 35 \mathrm{~d}$.

\section{A comparison of the $H p$ magnitudes with $V$ of the $U B V$ system}

For nine variables Hipparcos made simultaneous (within $1 \mathrm{~d}$ ) or nearly simultaneous (within $2 \mathrm{~d}$ ) observations with other photometric systems, viz. the Strömgren uvby-, Walraven $V B L U W$-, or Johnson $U B V$ photometric systems. Magnitudes in visual light obtained with the first two systems were transformed to the Johnson $V_{\mathrm{J}}$ of the $U B V$ system. The $H p$ passband is very wide and almost covers the three Johnson passbands, but has an effective wavelength very close to $V_{\mathrm{J}}$. Consequently, some difference between the two magnitude scales can be expected because of a possible dependence on the colours of the star. Small differences can be also expected due to light variations within $1 \mathrm{~d}-2 \mathrm{~d}(\leqq 0 \mathrm{~m} 05)$, although the program stars have in most cases periods longer than $10 \mathrm{~d}$. Such differences were discribed in ESA 1997, Vol. 1, Sect. 1.3 in a general comparison between $H p$ and $V_{\mathrm{J}}$ magnitudes. Table 1 lists the nine stars with their spectral type. The third and fourth column list the difference $\delta m=H p-V_{\mathrm{J}}$ and the number $(n)$ of observations used, respectively. The standard deviation $(\sigma)$ of $\delta m$ is of the order of a few 0.01 . These differences are mostly in good agreement with the curve shown in Fig. 1.3.4 of the above mentioned volume. The main difference is for HIP 67261, where a value of +0.15 would have been expected, but this can be attributed to the use of the wrong colour index in the Hipparcos data reductions.

A second method is to compare both magnitude scales with the aid of the mean magnitudes, at least if sufficient $V_{\mathrm{J}}$ data are known and if the star does not show a very long-time scale variability. Also when the range is large and the number of data is too low, no $\delta m$ value is given. Tables 2 and 3 (to be discussed in Sect. 5 ) lists these $\delta m$ values. 
Table 3. Particulars of the program stars in the Galaxy

\begin{tabular}{|c|c|c|c|c|c|c|c|c|c|c|}
\hline \multicolumn{3}{|c|}{ Star } & Sp & $H p$ & $\overline{V_{\mathrm{J}}}$ & $\delta m$ & max range & $\log T_{\text {eff }}$ & $M_{\mathrm{bol}}$ & Remarks \\
\hline HIP & HD & Name & & & & & & & & \\
\hline$\overline{37444}$ & 62150 & & B4 I & 7.79 & 7.68 & 0.11 & 0.12 & $4.17^{a}$ & $-8.0^{a}$ & \\
\hline 42570 & 74180 & & F2 Ia & 3.93 & 3.84 & $0.09^{b}$ & $0.06^{c}$ & $3.85^{d}$ & $-8.8^{d}$ & \\
\hline 45467 & 80077 & & $\mathrm{~B} 2 / 3 \mathrm{Ia}^{+}$ & 7.57 & 7.58 & $-0.01^{b}$ & 0.20 & $4.28^{e}$ & $-11.1^{e}$ & LBV $?^{e}$ \\
\hline 53444 & $94878^{g}$ & GG Car ${ }^{g}$ & $\mathrm{Be}$ & 8.80 & 8.72 & 0.08 & $0.2^{f}$ & & & \\
\hline $54463^{i}$ & 96918 & V382 Car & ${\mathrm{G} 0 \mathrm{Ia}^{+}}^{+}$ & 4.09 & 3.95 & $0.11^{b}$ & 0.12 & $3.72^{h}$ & $-9.0^{h}$ & \\
\hline 67261 & 119796 & V766 Cen & $\mathrm{G}_{8} \mathrm{Iae}^{+}-\mathrm{K} 3 \mathrm{Ia}^{+}$ & 6.70 & 6.8 & $b$ & 1 & $3.59^{j}$ & $-10.8^{j}$ & HR 5171A \\
\hline 80782 & 148379 & QU Nor & B2 Iab & 5.44 & 5.36 & 0.08 & 0.11 & $4.26^{k}$ & $-9.0^{k}$ & \\
\hline 89956 & 168607 & V4029 Sgr & $\mathrm{B} 9 \mathrm{Ia}^{+}$ & 8.23 & 8.16 & $0.07^{b}$ & 0.31 & $3.97^{l}$ & $-8.7^{l}$ & LBV \\
\hline 89963 & 168625 & V4030 Sgr & $\mathrm{B} 5 / 8 \mathrm{Ia}^{+}$ & 8.48 & 8.39 & $0.09^{b}$ & 0.19 & $4.08^{l}$ & $-8.6^{l}$ & $\mathrm{LBV} ?^{m}$ \\
\hline
\end{tabular}

Remarks to Table 3:

${ }^{a}$ Averages from Table 1 in van Genderen (1985).

${ }^{c}$ Not reliable (Steemers \& van Genderen 1986).

e Steemers \& van Genderen (1986), also Carpay et al. (1991).

${ }^{g}$ See Gosset et al. $(1984,1985)$.

${ }^{i}$ Characteristics of variability discovered by Hipparcos.

${ }^{k}$ van Genderen (1986).

${ }^{m}$ Nota et al. (1996).

\section{Some physical parameters of the program stars}

Table 2 lists the SMC- and LMC objects, and Table 3 the galactic objects, respectively, identified by their numbers, the spectral type, for the SMC and LMC only the galactic foreground reddening (see further), the adopted reddening, the mean $H p$ and $V_{\mathrm{J}}$ magnitude, $\delta m=H p-V_{\mathrm{J}}$, the maximum range ever observed (only the micro variations if an eclipse, or an SD (S Dor)-type variability is involved), $\log T_{\text {eff }}$ (for most of the stars derived from the spectral type calibration of de Jager \& Nieuwenhuijzen $1987)$ and at last $M_{\mathrm{bol}}$. For the SMC and LMC $M_{\mathrm{bol}}$ is in most cases based on the distance moduli 19.1 and 18.6, respectively, or are based on an indirect method. For the galactic objects $M_{\text {bol }}$ is taken from various references.

The galactic foreground reddening $E(B-V)_{\mathrm{J}}$ of the Magellanic Cloud objects were extracted from Figs. 7b, c of Schwering \& Israel (1991). They used the HI distribution to separate the galactic infrared emission in front of the Clouds themselves and constructed maps of the smoothed galactic foreground colour excess $E(B-V)_{\mathrm{J}}$ distribution towards the Clouds. It should be emphasized that apart from local fluctuations, smaller than the resolution of $48^{\prime}$, these values are only a lower limit. The reddening in the Clouds should be added, but in most cases it is unknown. If a literature value for the total reddening is known, but lower than the galactic foreground reddening, the latter is used for the determination of $M_{\mathrm{bol}}$ instead of the literature value. If no literature value is known then it is derived from the spectrum $/(B-V)_{\mathrm{J}}$ relationship (Schmidt-Kaler 1982) and the observed $(B-V)_{\mathrm{J}}$. If this

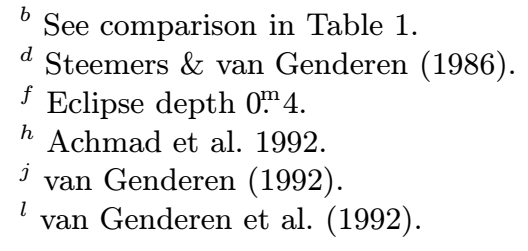

reddening is lower than the galactic foreground reddening, the latter is used. Therefore, in many cases $M_{\text {bol }}$ is presumably a lower limit.

\section{References}

Achmad L., Lamers H.J.G.L.M., Nieuwenhuijzen H., et al., 1992, A\&A 259, 600

Ardeberg A., Brunet J.P., Maurice E., et al., 1972, A\&AS 6, 249

Berdnikov L.N., Turner D.G., 1995, IBVS 4202

Burki G., 1976, A\&A 65, 357

Burki G., 1987, Proc. Workshop Instabilities in Luminous Early Type Stars, Lamers H.J.G.L.M., de Loore C.W.H., (eds.). Reidel, p. 23

Carpay J., de Jager C., Nieuwenhuijzen H., et al., 1989, A\&A 216, 143

Carpay J., de Jager C., Nieuwenhuijzen H., et al., 1991, A\&A 248,475

Eggen O.J., 1983, AJ 88, 1458

ESA, The Hipparcos and Tycho Catalogues, 1997, ESA SP1200

Feast M.W., Thackeray A.D., Wesselink A.J., 1969, MNRAS 121,337

Feinstein A., 1968, Z.f.Ap. 68, 29

van Genderen A.M., 1979, A\&AS 38, 381

van Genderen A.M., 1985, A\&A 151, 349

van Genderen A.M., 1986, A\&A 157, 163

van Genderen A.M., 1989, A\&A 208, 135

van Genderen A.M., 1991, ESO Workshop on Rapid Variability of OB-Stars: Nature and Diagnostic Value, Baade D. (ed.), p. 117

van Genderen A.M., 1992, A\&A 257, 177 
van Genderen A.M., Alphenaar P., van der Bij M.D.P., et al., 1985, A\&AS 61, 213

van Genderen A.M., van den Bosch F.C., Dessing F., et al., 1992, A\&A 264, 88

van Genderen A.M., van Driel W., Greidanus H., 1986, A\&A 155,72

van Genderen A.M., de Groot M., Thé P.S., 1983, A\&A 117, 53

van Genderen A.M., de Groot M., Sterken C., 1997b, A\&AS 124,517

van Genderen A.M., Hadiyanto Nitihardjo G., 1989, A\&AS 79, 401

van Genderen A.M., van Leeuwen F., Brand J., 1982, A\&AS 47,591

van Genderen A.M., Sterken C., de Groot M., 1997a, A\&A 318,81

Gosset E., Surdej J., Swings J.P., 1984, A\&AS 55, 411

Gosset E., Hutsemékers D., Surdej J., et al., 1985, A\&A 153, 71

Grieve G.R., Madore B.F., 1986, ApJS 62, 451

Grieve G.R., Madore B.F., Welch D.L., 1985, ApJ 294, 513

Houk N., 1978, Michigan Catalogue of Spectral Types 2

de Jager C., Mulder P.S., Kondo Y., 1984, A\&A 141, 304

de Jager C., Nieuwenhuijzen H., 1987, A\&A 177, 217

Kholopov N., Samus' N.N., Frolov M.S., et al., 1985, Gen. Cat. Variable Stars, Moscow, Nauta

van Leeuwen F., Evans D.W., van Leeuwen-Toczko M.B., 1997, in: Statistical Challenges in Modern Astronomy II, Feigelson E. and Babu G.J. (eds.) (in press)

Manfroid J., Sterken C., Cunow B., et al., 1994, Third Cat. Stars Measured in the LTPV Project (1990-1992), A\&AS
109,329

Mantagazza L., 1992, A\&A 265, 527

Nieuwenhuijzen H., de Jager C., Groth H.G., 1997, A\&A (in preparation)

Nota A., Pasquali A., Clampin M., et al., 1996, Proc. 33rd Liège Int. Astrophys. Coll., Wolf-Rayet Stars in the Framework of Stellar Evolution, Vreux J.-M., Detal A., Fraipont-Caro D. et al. (eds.), p. 453

Prinja R.K., Schild H., 1991, A\&A 249, 428

Rosendhal J.D., Snowden M.S., 1971, ApJ 169, 281

Schmidt-Kaler Th., 1982, Landolt-Börnstein Gruppe IV, Band 2

Schwering P.B.W., Israel F.P., 1991, A\&A 246, 231

Stahl O., Wolf B., Zickgraf F.J., 1987, A\&A 184, 193

Steemers W.J.G., van Genderen A.M., 1986, A\&A 154, 308

Stellingwerf R.F., 1978, ApJ 224, 953

Sterken C., 1977, A\&A 57, 361

Sterken C., 1983, The Messenger 33, 10

Sterken C., Manfroid J., Anton K., et al., 1993, Second Cat. Stars Measured in the LTPV Project (1986-1990) A\&AS 102,79

Sterken C., Manfroid J., Beele D., et al., 1995, Fourth Cat. Stars Measured in the LTPV Project (1992-1994) A\&AS 113,31

Sterken C., de Groot M., van Genderen A.M., 1997, A\&A 326, 640

Sterken C., de Groot M., van Genderen A.M., 1998, A\&A (in preparation)

Szeifert Th., Stahl O., Wolf B., et al., 1993, A\&A 280, 508

Wolf B., Stahl O., de Groot M., et al., 1981, A\&A 99, 351 